\title{
The Mantle Cell Lymphoma International Prognostic Index: Does it work in routine practice?
}

\author{
S.Y. LIM, J.M. HORSMAN and B.W. HANCOCK \\ Academic Unit of Clinical Oncology, Weston Park Hospital, Sheffield S10 2SJ, UK
}

Received June 3, 2009; Accepted September 28, 2009

DOI: $10.3892 /$ ol_00000034

\begin{abstract}
The Mantle Cell Lymphoma International Prognostic Index (MIPI) combines four factors to differentiate low-, intermediate- and high-risk prognostic groups in advanced mantle cell lymphoma using data from patients treated in clinical trials. To evaluate its use in routine practice, we applied the simplified index retrospectively to 50 consecutive new patients attending our lymphoma service. In the log-rank and multiple comparison statistical tests there was favorable differentiation between survival curves, and particularly between the high- and low-risk groups. We concluded that the MIPI is of value in routine lymphoma practice.
\end{abstract}

\section{Introduction}

Mantle cell lymphoma (MCL) is an uncommon yet distinct entity accounting for approximately 3-6\% of all non-Hodgkin lymphoma cases (1-3). It was added to the Revised EuropeanAmerican Classification of Lymphoid Neoplasms in 1994, two years after the name was coined by Banks and colleagues (4). It is characterized by a poor patient prognosis with a reported median overall survival (OS) of only 30-43 months $(1,2,5)$.

In 2008, Hoster et al developed the Mantle Cell Lymphoma International Prognostic Index (MIPI) as the first prognostic index specific to patients with advanced stage MCL (6). The prognostic index is based on four independent factors: age, performance status, lactate dehydrogenase (LDH) and leukocyte count. Cell proliferation (Ki-67) was exploratively analyzed as an important biological marker and showed strong additional prognostic relevance. Using data from patients treated in clinical trials, the authors described the simplified prognostic index, which yielded high concordance (weighted $\kappa=0.79)$ and good separation of the overall survival curves. However, no independent study is currently available to

Correspondence to: Professor B.W. Hancock, YCR Academic Unit of Clinical Oncology, Weston Park Hospital, Whitham Road, Sheffield S10 2SJ, UK

E-mail: b.w.hancock@sheffield.ac.uk

Key words: Mantle Cell Lymphoma International Prognostic Index validate this index in routine clinical practice. We, therefore, retrospectively applied MIPI to our MCL patients.

\section{Materials and methods}

This was a retrospective study. All consecutive MCL patients treated in Weston Park Hospital from 1981 to 2008 were included. The patient population was obtained from a dedicated Lymphoma Database.

The four prognostic factors assessed were: age, performance status, LDH and leukocyte count. Simplified MIPI was applied to stratify patients into low- (score 0-3), intermediate(score 4-5) or high-risk (score 6-11) groups (Table I). Survival was calculated from the date of diagnosis. The Kaplan-Meier survival curves were calculated for the three risk groups and compared using the log-rank test for trend.

\section{Results}

Seventy-six patients attended from January 1981 to July 2008. Only 50 patients had complete data for MIPI scoring. The median age was 64 years. Thirty-eight of the patients $(66 \%)$ were males.

According to the simplified MIPI scoring system, 11 patients $(22 \%)$ were in low-, $23(46 \%)$ in intermediate- and 16 patients $(32 \%)$ in high-risk groups.

There was a statistically significant trend in survival across the three groups ( $\mathrm{p}=0.013$ ), with median survival in the low-, intermediate- and high-risk groups being 9.89, 3.85 and 2.81 years, respectively (Fig. 1). Each risk group was compared with each of the other two using the Bonferroni corrected threshold to adjust for multiple comparisons. Survival time in the high-risk group was statistically significantly less than in the low-risk group $(\mathrm{p}=0.0112)$.

\section{Discussion}

Until 2007, there was no generally established prognostic index for patients with MCL. The International Prognostic Index (IPI) and Follicular Lymphoma International Prognostic Index (FLIPI) had been developed for diffuse large B-cell (DCBCL) and follicular lymphoma patients, respectively. IPI and FLIPI showed poor separation of survival curves for MCL patients (6). The IPI score classifies most patients in the two intermediate-risk groups and does not separate the outcomes. 
Table I. The simplified Mantle Cell Lymphoma International Prognostic Index was used to stratify patients into risk groups according to the four prognostic factors.

\begin{tabular}{lcccc}
\hline Points & Age & ECOG PS & LDH (ULN) & WBC (10 $/ 1)$ \\
\hline 0 & $<50$ & $0-1$ & $<0.67$ & $<6.700$ \\
1 & $50-59$ & - & $0.67-0.99$ & $6.700-9.999$ \\
2 & $60-69$ & $2-4$ & $1.0-1.49$ & $10.000-14.999$ \\
3 & $\geq 70$ & - & $\geq 1.5$ & $\geq 15.000$ \\
\hline
\end{tabular}

ECOG PS, Eastern Cooperative Oncology Group Performance Status; LDH (ULN), lactate dehydrogenase (upper limit of the normal range); WBC, white blood cell (leukocyte) count. Total point score: 0-3, low; 4-5, intermediate and 6-11, high risk.

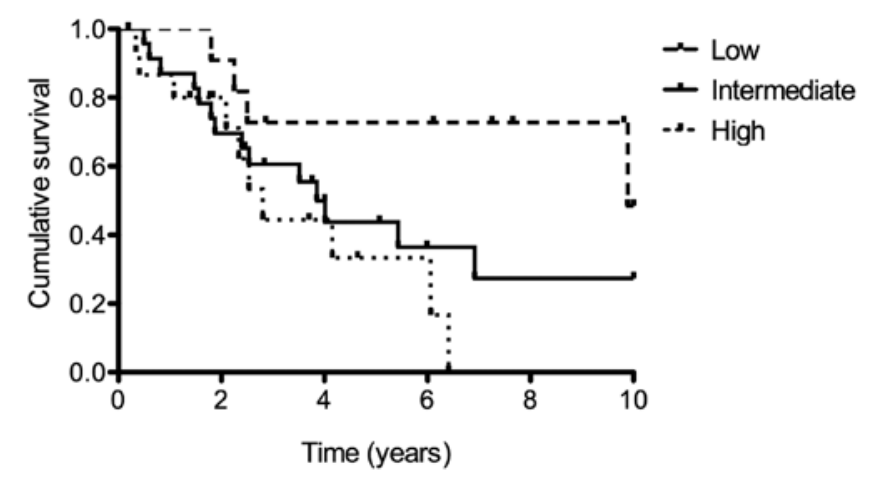

Figure 1. Cumulative patient survival in the low-, intermediate- and high-risk patient groups.

The FLIPI score places most patients into the high-risk group and does not separate low from intermediate in outcome (7). MIPI, derived using data of 455 advanced stage patients treated within three clinical trials, proved to be a suitable index for MCL patients (7).

We retrospectively applied the simplified MIPI to all of the consecutive patients (for whom full data were available) referred to our lymphoma service. MIPI proved a valuable guide to prognosis with a significant trend for difference between low-, intermediate- and high-risk groups, but in particular low- and high-risk.

Conventional non-trial treatment for younger patients with MCL is anthracycline-containing (CHOP-like) chemotherapy, which in responding patients proceeds to consolidative intensive chemotherapy with autologous stem cell rescue (recently with the addition of rituximab). In our series only 7 patients went on to have high-dose therapy. As expected, in this selected group, survival was favorable (mean overall survival, 97 months; range, 49-132). Four patients were in the best-risk group by MIPI. Only 1 patient was in the high-risk group and he had the shortest survival. On the other hand, Martin et al reported that it may be possible to select certain asymptomatic patients with MCL for deferred initial treatment ('watch and wait') (8). When we evaluated our patients there were 3 who had little or no previous treatment and had better than expected survival; the 3 patients had low-risk MIPIs.
Evidently there are factors at work other than those comprising MIPI, and a search for these continues. In the interim, the index provides us with a reasonable guide to prognosis which works in unselected cases of mantle cell lymphoma presenting to an orthodox lymphoma service.

\section{References}

1. Andersen NS, Jensen MK, de Nully Brown P and Geisler CH: A Danish population-based analysis of 105 mantle cell lymphoma patients: incidences, clinical features, response, survival and prognostic factors. Eur J Cancer 38: 401-408, 2002.

2. Velders GA, Kluin-Nelemans JC, de Boer CJ, et al: Mantle cell lymphoma: a population-based clinical study. J Clin Oncol 14: 1269-1274, 1996.

3. A clinical evaluation of the International Lymphoma Study Group Classification Project. Blood 89: 3909-3918, 1997.

4. Banks PM, Chan J, Cleary ML, et al: Mantle cell lymphoma. A proposal for unification of morphologic, immunologic and molecular data. Am J Surg Pathol 16: 637-640, 1993.

5. Argatoff LH, Connors JM, Klasa RJ, et al: Mantle cell lymphoma: a clinicopathologic study of 80 cases. Blood 89: 2067-2078, 1997.

6. Hoster E, Dreyling M, Klapper W, et al: A new prognostic index (MIPI) for patients with advanced-stage mantle cell lymphoma. Blood 111: 558-565, 2008.

7. Smith MR: Mantle cell lymphoma: advances in biology and therapy. Curr Opin Haematol 15: 415-421, 2008.

8. Martin P, Chadburn A, Christos P, et al: Outcome of deferred initial therapy in mantle cell lymphoma. J Clin Oncol 27: 1209-1213, 2009. 